\title{
1 Rate equations for sodium catalyzed 2 amorphous silica dissolution
}

3 J. Donald Rimstidt"a, Yilun Zhangb, and Chen Zhu ${ }^{\mathrm{b}}$

4 aDepartment of Geosciences, Virginia Tech, Blacksburg, VA 24061, USA

5 bDepartment of Geological Sciences, Indiana University, Bloomington, IN 47405, USA

6 Abstract-Newly measured amorphous silica dissolution rate data were combined with data from

7 the literature to produce an equation that predicts the dissolution flux $\left(U, \mathrm{~mol} / \mathrm{m}^{2} \mathrm{sec}\right)$ of amorphous

8 silica as a function of temperature $(T, K)$, sodium concentration $\left(m_{\mathrm{Na}+}\right.$, molal $)$, and hydrogen ion

9 activity $\left(a_{\mathrm{H}+}\right)$.

$10 \quad J_{N a}=14.62\left(10^{\left(\frac{77400}{2.303 R}\right)\left(\frac{1}{T}\right)}\right)+8.95\left(10^{\left(\frac{77700}{2.303 R}\right)\left(\frac{1}{T}\right)}\right)\left(\frac{m_{N a^{+}}^{0.490}}{a_{H^{+}}^{0.377}}\right)$

11 The same data fit equally well to an equation that expresses the dissolution flux as a function of

12 temperature, sodium concentration, and hydroxide ion activity $\left(a_{\mathrm{OH}}\right)$.

$13 J=14.62\left(10^{\left(\frac{77400}{2.303 R}\right)\left(\frac{1}{T}\right)}\right)+1.73 \times 10^{6}\left(10^{\left(\frac{77700}{2.303 R}\right)\left(\frac{1}{T}\right)}\right) m_{\mathrm{Na}^{+}}^{0.490} a_{\mathrm{OH}}^{0.377}$

14 These equations have the same form as those for quartz (Rimstidt 2015) and show that sodium ions

15 have the same catalytic effect on amorphous silica dissolution as they do for quartz.

\section{1. Introduction}

17 Amorphous silica phases, and closely related natural and synthetic glasses, are common in nature

18 as well as in technology. These amorphous phases are more soluble than quartz so they dissolve

19 more rapidly (Rimstdit and Barnes, 1980). Like quartz, amorphous silica dissolves faster at high pH

\footnotetext{
${ }^{*}$ Corresponding author.

E-mail address: jdr02@vt.edu
} 
by a process that is catalyzed by cations. This means that the weathering and diagenesis of opaline

21 biogenic silica (e.g. phytoliths, diatoms, sponge spicules) is significantly affected by solution

22 composition. In addition, it is possible that the weathering and transformation rates of natural and

23 synthetic glasses are also catalyzed by dissolved cations. In this study we combined some newly

24 measured amorphous silica dissolution rate data with data from the literature to develop rate

25 equations for amorphous silica dissolution as a function of $\mathrm{pH}$, sodium concentration $\left(m_{\mathrm{Na}}\right)$ and

26 temperature (T). Although these rate equations are only valid for pure silica, they serve as a useful

27 baseline to help us understand the dissolution rates of silica rich natural and synthetic phases.

28 Silica dissolves in water by releasing silicic acid into solution.

$29 \mathrm{SiO}_{2}(s)+2 \mathrm{H}_{2} \mathrm{O}=\mathrm{H}_{4} \mathrm{SiO}_{4}(a q)$

At high $\mathrm{pH}$ some of the silicic acid ionizes.

$31 \mathrm{H}_{4} \mathrm{SiO}_{4}(a q)=\mathrm{H}^{+}+\mathrm{H}_{3} \mathrm{SiO}_{4}^{-}$

32 So there is a second dissolution reaction that occurs in parallel with reaction (1).

$33 \mathrm{SiO}_{2}(s)+2 \mathrm{H}_{2} \mathrm{O}=\mathrm{H}^{+}+\mathrm{H}_{3} \mathrm{SiO}_{4}$

34 However, combining equation (3) with the equation for the ionization of water $\left(\mathrm{H}_{2} \mathrm{O}=\mathrm{H}^{+}+\mathrm{OH}^{-}\right)$

35 allows this reaction to also be written in terms of hydroxide ion.

$36 \mathrm{SiO}_{2}(s)+\mathrm{H}_{2} \mathrm{O}+\mathrm{OH}^{-}=\mathrm{H}_{3} \mathrm{SiO}_{4}^{-}$

37 Rimstidt (2015) found that quartz dissolution rates were fit equally well by a pH-based rate 38 equation and a pOH-based rate equation. The $\mathrm{pH}$-based equation implies that the reaction is 39 inhibited by hydrogen ions. This is consistent with rate equations based on surface speciation such 40 as those in Bickmore et al. (2008). The pOH-based equation suggests that hydroxide ions act as 41 nucleophilic reagents attacking Si centers. Although the authors of this paper prefer this 42 explanation because it is consistent with the Lewis acid base mechanism highlighted in the 43 quantum mechanical model of Xiao and Lasaga (1996), we realize that it cannot be justified by the 44 regression model alone. Appendix 2 in Rimstidt (2015) lists additional papers that discuss these 45 and other ideas about silica dissolution reaction mechanisms. 
47 Altogether 215 dissolution rate data were tabulated from three literature sources (Fraysse et al.,

48 2006; Icenhower and Dove, 2000; Wirth, 1980). We did not use data from studies where the reacting solutions contained pH buffers (e.g. Mazer and Walther, 1994) nor did we use data from studies that used silica samples with surface coatings such as 'Monospher', which is coated with sulfonic acid functional groups (Seidel et al., 1997), or silica diatoms, which have natural organic coatings (Greenwood et al., 2005). The rate data that we used are listed in the Supplementary Information data table along with the temperature, $\mathrm{pH}$ and the sodium concentration for the experiments. All rates are normalized to BET surface area. Quench $\mathrm{pH}$ values $\mathrm{pH}$ at room temperature) were used to develop the regression models. The actual $\mathrm{pH}$ of the solution at temperature was somewhat different. However, fits of data where the $\mathrm{pH}$ was adjusted for the effect of temperature produced regression parameters and $\mathrm{R}^{2}$ values that were nearly the same as those based on quench $\mathrm{pH}$. Therefore, we chose to fit the data using the quench $\mathrm{pH}$ in order to avoid introducing unnecessary complexity into the models.

There were very few rate measurements at high $\mathrm{pH}$ and moderate sodium concentration so 15 additional rate measurements were made in order to better constrain the rate model. These experiment were carried out at $0 \leq \mathrm{T} \leq 50^{\circ} \mathrm{C}, 5.3 \leq \mathrm{pH} \leq 10.4$ and $0 \leq m_{\mathrm{Na}} \leq 1.0$. The experiments used fused quartz wool, which is pure $\mathrm{SiO}_{2}$ glass (amorphous silica), from Technical Glass Product, Inc. The diameters of the quartz wool fibers ranged from 6 to $9 \mu \mathrm{m}$. The BET surface area of this material was $1.39 \mathrm{~m}^{2} / \mathrm{g}$ (measured by Micromeritics, Inc.). The geometric surface area was estimated to be $0.20 \mathrm{~m}^{2} / \mathrm{g}$. Solutions were prepared using reagent grade $\mathrm{NaCl}$ and the $\mathrm{pH}$ was adjusted using reagent grade $\mathrm{Na}_{2} \mathrm{CO}_{3}$ or $\mathrm{HCl}$. The quartz dissolution rate studies listed in the

68 Supplementary Information for Rimstidt (2015) shows good internal consistency among experiments conducted in chloride-, nitrate-, carbonate-, and sulfate-bearing solutions so we have assumed that silica dissolution rates are not affected by those anions.

71 The rates were measured using a mixed flow reactor with a recycle loop that ensured that the

72 solution in the reactor was well mixed (Figure 1). Two reactor sizes were used. One with a length of

$7310.3 \mathrm{~cm}$ and a diameter of diameter $1.9 \mathrm{~cm}\left(\right.$ volume $\left.=117 \mathrm{~cm}^{3}\right)$ was loosely packed with $5 \mathrm{~g}$ of

74 quartz wool. The other, used for higher temperatures and sodium concentrations, with a length of

$754.0 \mathrm{~cm}$ and a diameter of diameter $1.9 \mathrm{~cm}\left(\right.$ volume $\left.=45 \mathrm{~cm}^{3}\right)$ was loosely packed with $0.5 \mathrm{~g}$ of 
quartz wool. The fused quartz was used as obtained with no pretreatment. In order to ensure that this reactor behaved as an ideal mixed flow reactor, the feed pump was set to a low flow rate (3 to $50 \mathrm{~mL} / \mathrm{hr})$ and the recycle pump was set to a much higher flow rate $(\sim 1000 \mathrm{~mL} / \mathrm{hr})$ so that the solution passed over the glass wool many times during its residence in the reactor. Each experiment was run until the silica concentration varied by less than $10 \%$ from day to day. This

81 typically occurred seven to ten days after the beginning of the experiment.

82

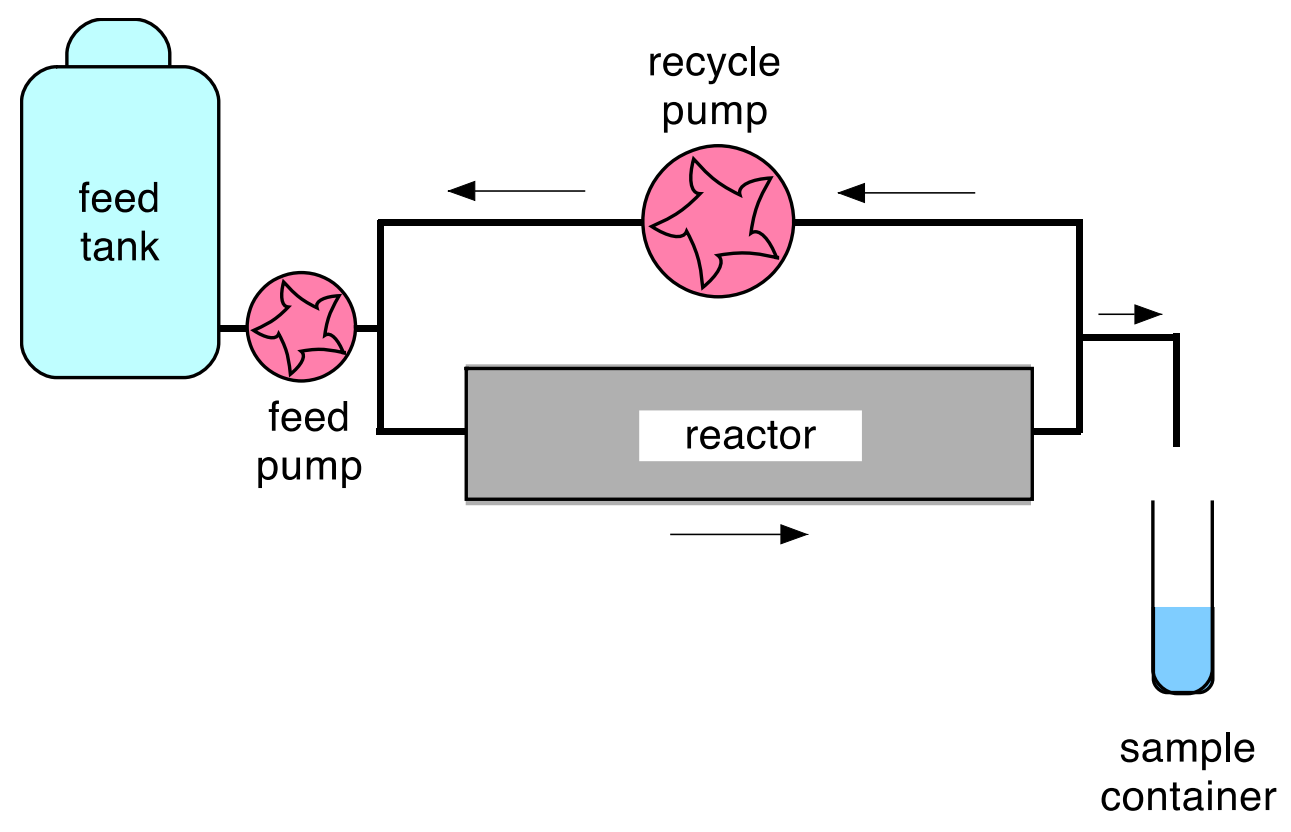

84 Figure 1. Schematic illustration of the externally recycled mixed-flow reactor used to measure the 85 dissolution rate of the quartz wool.

86 Before each experiment, the column containing the fused quartz was partly filled with deionized

87 water. It was then connected to the feed pump, which was operated at a slow flow rate until all of

88 the air bubbles were forced from the reactor. Then the recycle pump was run in the reverse

89 direction until air bubbles were cleared from the recycle loop. When the system was fully filled with

90 water the recycle pump was turned on in the forward direction and the feed pump inlet was

91 connected to the tank containing the experimental solution.

92 Once per day about $10 \mathrm{~mL}$ of effluent was collected into a weighed, acid-washed $15 \mathrm{~mL}$ centrifuge

93 tube. The flow rate was determined from the change in mass of the tube and the time of sample 
94 collection. The $\mathrm{pH}$ of the effluent was measured immediately after sample collection. Samples were 95 stored at $4^{\circ} \mathrm{C}$ until analysis. The silicon concentration was determined using the silicon molybdate 96 blue colorimetric method. There was no dissolved silica in the feed solution so the dissolution flux 97 was found from the product of the effluent silica concentration and flow rate divided by the BET 98 surface area of the quartz wool.

$99 \quad J=\frac{m_{S i} Q}{A}$

100 Table 1 gives a summary of the notation and units used in this paper. The approach used to develop 101 the rate equations the same as described in Rimstidt (2015). In the absence of sodium, the 102 dissolution flux $\left(U_{0}\right)$ from reaction (1) is only a function of temperature. A second reaction path is 103 responsible for the sodium catalyzed dissolution flux $\left(U_{\mathrm{Na}}\right)$. The rate of this second reaction is a 104 function of temperature, $\mathrm{pH}$, and sodium concentration. The overall dissolution flux is the sum of 105 these two parallel reactions.

$106 J=J_{o}+J_{N a}$

107 This required a two-step fitting process. First the logarithm of the dissolution flux of amorphous 108 silica in sodium absent solutions $\left(\log J_{0}\right)$ was fit as a function of $1 / T$.

$109 \log J_{o}=\log A+\frac{E_{a}}{2.303 R}\left(\frac{1}{T}\right)$

110 Then the dissolution flux predicted by this rate equation was subtracted from the dissolution flux

111 for experiments where the solutions contained dissolved sodium. The remaining flux $\left(U_{\mathrm{Na}}\right)$ was

112 attributed to a reaction that involves the catalytic effect of sodium. The logarithm of this difference 113 was fit as a function of $\mathrm{pH}(\mathrm{or} \mathrm{pOH}), m_{\mathrm{Na}}$, and $1 / T$.

$114 \log J_{N a}=\log A+\frac{E_{a}}{2.303 R}\left(\frac{1}{T}\right)+n_{H^{+}} p H+n_{N a^{+}} \log m_{N a^{+}}$

115 or

$116 \log J_{\mathrm{Na}}=\log A+\frac{E_{a}}{2.303 R}\left(\frac{1}{T}\right)+n_{\mathrm{OH}} \mathrm{pOH}+n_{\mathrm{Na}^{+}} \log m_{\mathrm{Na}^{+}}$ 
117 The antilog transforms of the two fitted equations (either (7) and (8) or (7) and (9)) were added to 118 get an equation that predicts amorphous silica rates $\mathrm{pH}$ (or $\mathrm{pOH}$ ), $m_{\mathrm{Na}}$, and $T$

119 Table 1. Notation used in this paper. Because the rate that silica is released from the amorphous 120 silica surface to the solution has units of flux that rate is called the dissolution flux $\left(\mathrm{J}, \mathrm{mol} / \mathrm{m}^{2} \mathrm{sec}\right)$.

\begin{tabular}{|c|l|}
\hline$A$ & pre-exponential in Arrhenius equation, $\mathrm{mol} / \mathrm{m}^{2} \mathrm{sec}$ \\
$a_{i}$ & activity of species $i$ \\
$E_{a}$ & activation energy, $\mathrm{kJ} / \mathrm{mol}$ \\
$J$ & overall dissolution flux $\left(J=J_{\mathrm{o}}+J_{\mathrm{Na}}\right), \mathrm{mol} / \mathrm{m}^{2} \mathrm{sec}$ \\
$J_{\mathrm{Na}}$ & sodium catalyzed dissolution flux, $\mathrm{mol} / \mathrm{m}^{2} \mathrm{sec}$ \\
$J_{\mathrm{o}}$ & dissolution flux in sodium absent solutions, $\mathrm{mol} / \mathrm{m}^{2} \mathrm{sec}$ \\
$m_{i}$ & concentration of species $i$, molal \\
$n_{i}$ & reaction order for species $i$ \\
$\mathrm{pH}$ & $\mathrm{pH}\left(=-\log a_{\mathrm{H}}\right)$ at $25^{\circ} \mathrm{C}$ \\
$\mathrm{pOH}$ & pOH $\left(=-\log a_{O H}\right)$ at $25^{\circ} \mathrm{C}$ \\
$Q$ & flow rate, $\mathrm{kg} / \mathrm{sec}$ \\
$R$ & gas constant, $8.314 \mathrm{~J} / \mathrm{mol} \mathrm{K}$ \\
$T$ & temperature, ${ }^{\circ} \mathrm{C}$ or $\mathrm{K}$ \\
\hline
\end{tabular}

122 The regression models were prepared using JMP10 (SAS Institute Inc.) statistical software. Partial 123 regression plots (Moya-Laraño and Corcobado, 2008; Velleman and Welsch, 1981), which show the 124 residuals of an independent variable versus the residuals of the dependent variable, were used to 125 visualize the contribution of each independent variable to the overall fit. The slope of the line 126 shown on those plots equals the regression coefficient for that variable. A steep slope means that 127 the independent variable strongly influences the dependent variable.

\section{Results}

129 The dissolution flux data from the experiments are listed in Table 2. These data were combined 130 with the literature data (see Supplemental Information) to produce a rate equation for amorphous 131 silica as a function of $\mathrm{pH}$, sodium concentration and temperature. During the modeling it was 132 discovered that the data from Table 4 in Icenhower and Dove (2000) (low temperature externally 133 recycled reactor experiments) consistently fell below the best-fit line, some by as much as one log 134 unit. We interpreted this to mean that there was a systematic problem with those data so we 135 removed them from the regression model. This left us with 36 data for the sodium-absent model and 164 data for the sodium-present model. 
137 Table 2. Amorphous silica dissolution flux values determined in this study.

\begin{tabular}{ccccc}
\hline experiment & $T,{ }^{\circ} \mathrm{C}$ & $\mathrm{pH}$ & $m_{\mathrm{Na}}$ molal & $\log J$ \\
\hline QW1 & 22 & 5.23 & 0.000 & -12.00 \\
QW2 & 22 & 7.74 & 0.001 & -11.96 \\
QW3 & 50 & 5.24 & 0.000 & -11.19 \\
QW5 & 22 & 9.80 & 0.001 & -10.40 \\
QW6 & 22 & 9.85 & 0.010 & -10.09 \\
QW7 & 22 & 10.04 & 0.100 & -10.00 \\
QW8 & 22 & 9.97 & 1.000 & -9.98 \\
QW9 & 50 & 9.92 & 0.001 & -9.37 \\
QW10 & 50 & 9.96 & 0.010 & -9.31 \\
QW11 & 50 & 10.00 & 0.100 & -9.15 \\
QW12 & 50 & 9.72 & 0.100 & -9.15 \\
QW13 & 0 & 9.81 & 0.001 & -11.36 \\
QW14 & 0 & 9.69 & 0.010 & -10.79 \\
QW15 & 0 & 9.54 & 0.100 & -11.72 \\
QW16 & 0 & 9.54 & 1.000 & -11.61 \\
\hline
\end{tabular}

138

139 Regression of all the data for the sodium absent experiments (Figure 2) produced the following 140 equation.

$141 \log J_{o}=1.165(0.171) \quad \frac{4042(65)}{T}$

$R^{2}=0.991 \quad n=36$

142 The numbers in parentheses following the fitted parameter represent one standard error for that

143 parameter. The activation energy derived from this fit is $77.4 \mathrm{~kJ} / \mathrm{mol}$. The antilog of this expression

144 gives an equation that predicts the amorphous silica dissolution flux as a function of temperature.

$145 J_{o}=14.62\left(10^{\left(\frac{77400}{2.303 R}\right)\left(\frac{1}{T}\right)}\right)$ 


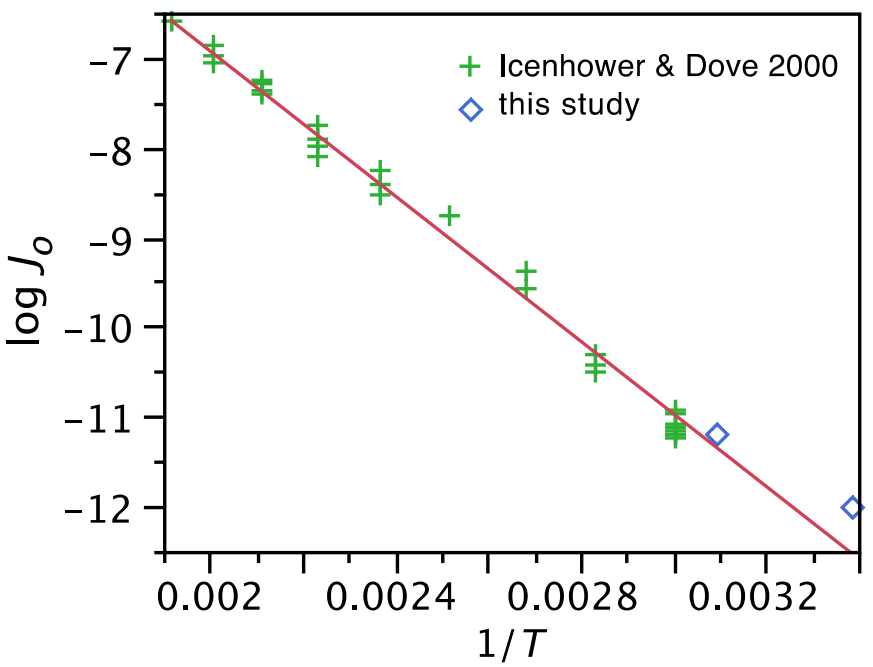

147 Figure 2. Graph of $\log J_{\mathrm{o}}$ versus $1 / T$ for experiments with no dissolved sodium.

148 The value of $J_{o}$ predicted by equation (11) was subtracted from the observed dissolution flux for 149 solutions that contained sodium to get the dissolution flux for the sodium catalyzed reaction. The 150 values of $\log J_{\mathrm{Na}}$ were then regressed against $1 / T, \mathrm{pH}$, and $\log m_{\mathrm{Na}}$ (Figure 3 ) to produce an equation 151 that describes the rate for the sodium catalyzed reaction.

$\log J_{N a}=0.952(0.129)+\frac{4057(51)}{T}+0.377(0.015) p H+0.490(0.030) \log m_{N a^{+}}$

$R^{2}=0.978 \quad n=164$

153 The numbers in parentheses following the fitted parameter represent one standard error for that 154 parameter. The antilog of this expression predicts the rate of the sodium-catalyzed reaction. The 155 activation energy for this reaction is $77.7 \mathrm{~kJ} / \mathrm{mol}$.

$156 \quad J_{N a}=8.95\left(10^{\left(\frac{77700}{2.303 R}\right)\left(\frac{1}{T}\right)}\right)\left(\frac{m_{N a^{+}}^{0.490}}{a_{H^{+}}^{0.377}}\right)$

157 Combining equation (12) with (13) gives an equation that predicts the overall amorphous silica 158 dissolution flux.

$159 J=14.62\left(10^{\left(\frac{77400}{2.303 R}\right)\left(\frac{1}{T}\right)}\right)+8.95\left(10^{\left(\frac{77700}{2.303 R}\right)\left(\frac{1}{T}\right)}\right)\left(\frac{m_{N a^{+}}^{0.490}}{a_{H^{+}}^{0.377}}\right)$ 

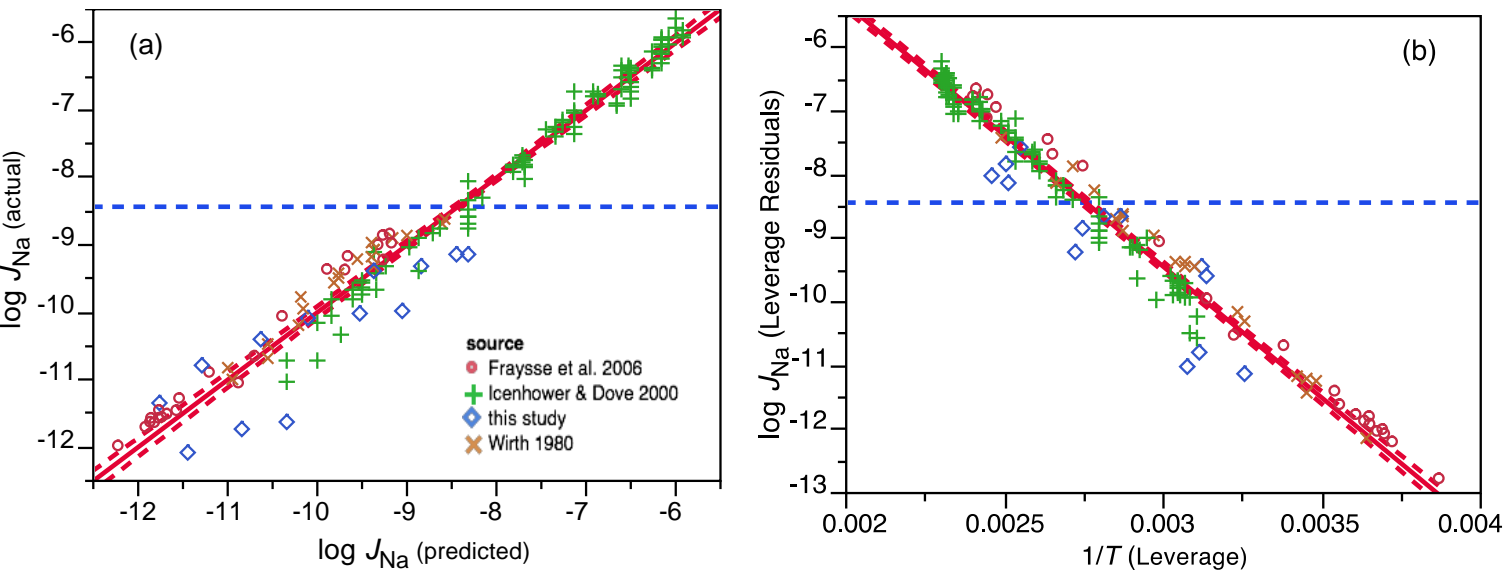

160
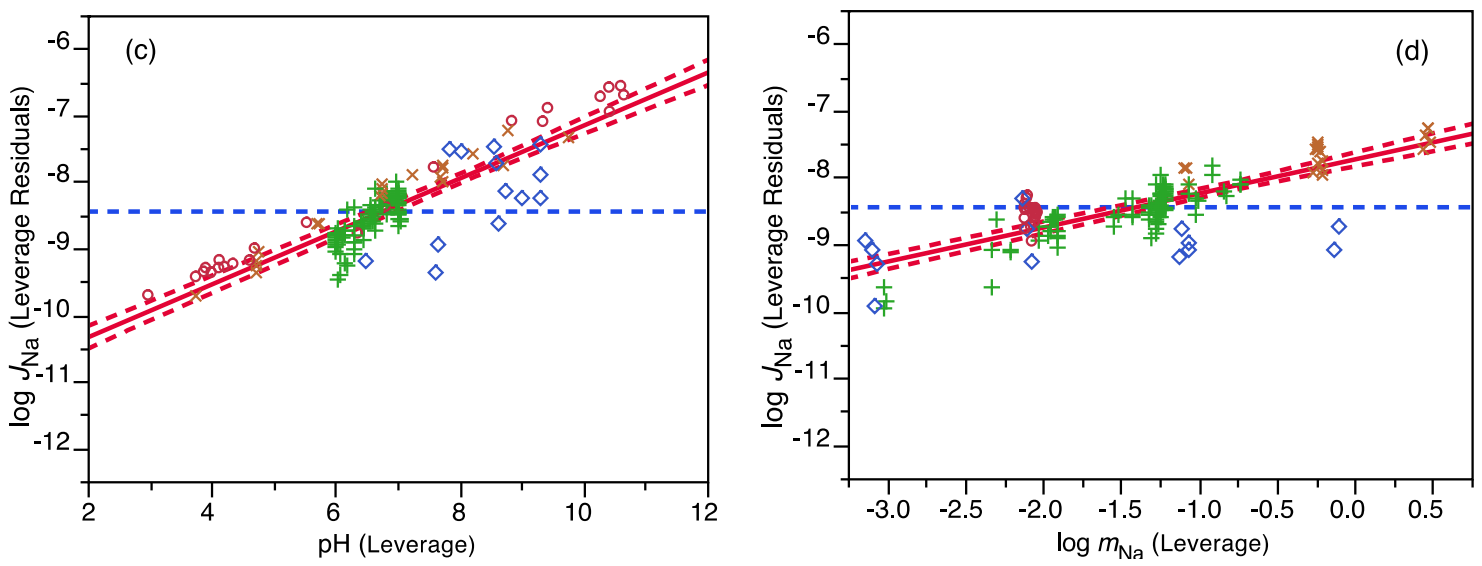

162 Figure 3. Fit of $\log J_{\mathrm{Na}}$ as a function of $\mathrm{pH}, \log m_{\mathrm{Na}}$, and $1 / T$. (a) Observed values of $\log J_{\mathrm{Na}}$ versus

163 predicted values of $\log J_{\text {Na. }}$ (b) Partial regression leverage plot for $1 / T$. (c) Partial regression

164 leverage plot for $\mathrm{pH}$. (d) Partial regression leverage plot for $\log m_{\mathrm{Na}}$.

165 Equation (14) predicts that rate of the sodium-catalyzed reaction in is inversely proportional to the

166 hydrogen ion activity, which implies that hydrogen ions inhibit the sodium catalyzed dissolution

167 reaction. An alternative explanation is that hydroxide ions participate in the activated complex

168 making the rate directly proportional to the hydroxide ion activity. We tested this possibility by

169 regressing $\log J_{\mathrm{Na}}$ against $1 / T, \mathrm{pOH}$, and $\log m_{\mathrm{Na}}$.

170

$\log J_{N a}=6.237(0.231)+\frac{4057(51)}{T}+0.490(0.030) \log m_{\mathrm{Na}^{+}} \quad 0.377(0.015) p O H$

$R^{2}=0.978 \quad n=164$ 
171 The numbers in parentheses represent one standard error of the fitted parameter. The patterns

172 found in the graphs for this regression model are identical to those for the pH-based model based

173 except the slope on the pOH partial regression plot is reversed. Because equations (13) and (15) fit

174 the data equally well, we cannot use the fitted rate equation to eliminate either hypothesis.

175 Taking the antilog of equation (15) and combining with equation (11) gives an equation that

176 predicts the overall amorphous silica dissolution flux in terms temperature, sodium concentration

177 and hydroxide activity.

\section{Discussion and Conclusions}

180 The rate equations given in this paper are suitable for modeling geochemical processes but they are

181 nothing more than empirical interpolations between the existing data. Because they are not based

182 on a fundamental model of the reaction mechanism we do not recommend extrapolating them

183 much beyond the experimental conditions. Furthermore, the fitting process may have been biased

184 by the clumped distribution of experimental conditions. The new experimental data reported in this

185 paper helps this problem somewhat by anchoring the data at high $\mathrm{pH}$ and low temperatures but the 186 model would be improved by data taken from a wider range of conditions. For example, there is

187 considerable evidence that amorphous silica and quartz dissolution rates increase below pH 3 but

188 we have not included any of those data in our model. As a result we recommend that the rate

189 equations be used only for $3<\mathrm{pH}<11$. Finally, our models are based on total sodium

190 concentrations and do not account for speciation effects or for the effects of other cations $\left(e . g . \mathrm{K}^{+}\right.$,

$191 \mathrm{Ca}^{2+}, \mathrm{Mg}^{2+}$, etc.). The dissolution flux values used to create the rate equations in this paper and in

192 Rimstidt (2015) are normalized to BET surface area measurements. Multiplying those values $\sim \times 5$

193 should give reasonable estimates of these dissolution flux values based on geometric surface area

194 (see the discussion about geometric and BET surface areas in Rimstidt et al., 2012).

195 Rimstidt (2015) showed that the effect of sodium concentration and pH on quartz dissolution rates

196 could be modeled equally well by multiple rate equations because of correlations among

197 independent variables used to construct those equations. In this study we showed that the rate 
equations based on $\mathrm{pH}$ and $\mathrm{pOH}$ fit the data equally well and we expect that rate equations based on

199 surface speciation, like those in Bickmore et al. (2008), would also fit the data quite well. As a result

200 it is not possible to infer a reaction mechanism from these rate equations alone.

201 The dissolution flux of amorphous silica as a function of $\mathrm{pH}$, contoured in sodium concentrations

202 (Figure 4) is essentially identical to the diagram for quartz (see Figure 7 in Rimstidt, 2015) except

203 that the contours are displaced to a higher value by about 1 log unit because amorphous silica

204 solubility is about ten times higher than for quartz. This is consistent with the idea that the reaction

205 mechanism is the same for both phases as assumed in the rate equations in Rimstdit and Barnes

206 (1980).

207 The rate equations in this paper provide a good baseline for understanding the dissolution rates of

208 related materials. The data used to produce the rate equations are from experiments that used

209 crushed fused quartz (Icenhower and Dove, 2000; Wirth and Gieskes, 1979), pyrolyzed silica

210 (Icenhower and Dove, 2000), plant phytoliths (Fraysse et al., 2006), and quartz wool (this study).

211 The dissolution rates of all of these materials appear to be concordant. The dissolution rates for

212 diatoms in seawater reported in Rickert et al. (2002) are somewhat scattered, no doubt because of

213 the challenge of obtaining reliable surface area values, but they are reasonably consistent with the

214 rate predicted for seawater sodium concentrations. Also, the rates for true seawater compositions

215 should be higher than the seawater sodium point on this figure due to the additional catalytic

216 effects of other cations. Figure 4 shows dissolution rates of nepheline, jadite, and albite glasses at

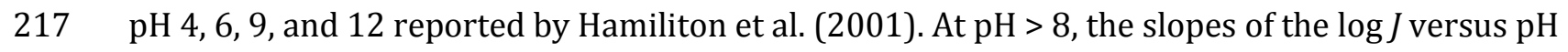

218 graphs are parallel to the sodium isopleths, which suggests that the bond breaking reactions that

219 release silica from these glasses might be the same as those that are responsible for amorphous

220 silica dissolution. 


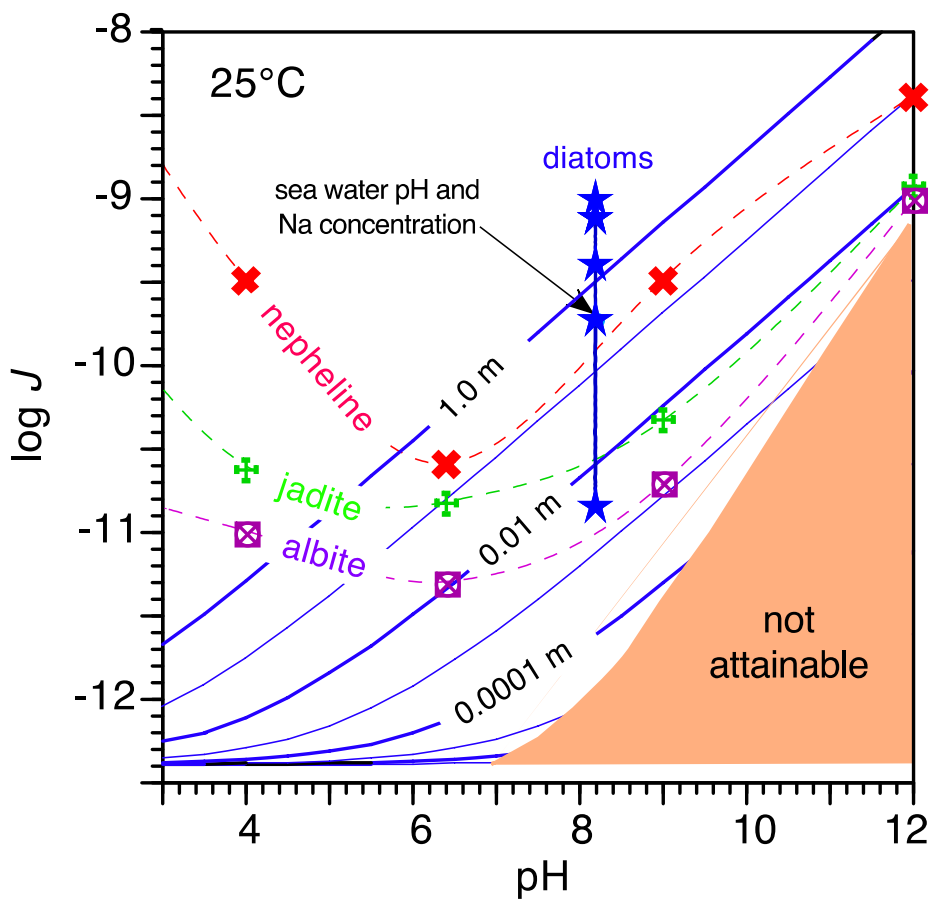

221

Figure 4. Predicted logarithm of the amorphous silica dissolution flux at $25^{\circ} \mathrm{C}$ as a function of $\mathrm{pH}$

223 contoured in $\mathrm{Na}^{+}$concentration, based on equation (14). The shaded area represents hydroxide

224 activities that are not attainable because sodium must be added to the solution along with the

225 hydroxide. Note that when no $\mathrm{Na}^{+}$is present the dissolution flux is independent of $\mathrm{pH}$. The star

226 symbols represent cleaned diatom dissolution rates in seawater reported by Rickert et al. (2002).

227 The other symbols show dissolution rates for nepheline, jadite, and albite glasses at pH 4, 6, 9, and

22812 reported by Hamiliton et al. (2001).

\section{Acknowledgments}

230 This research was supported by the National Science Foundation grant number EAR-1225733. The 231 authors thank Jonathan Icenhower and two anonymous reviewers for their helpful suggestions.

\section{References}

233 Bickmore, B.R., Wheeler, J.C., Bates, B., Nagy, K.L. and Eggett, D.L. (2008) Reaction 234 pathways for quartz dissolution determined by statistical and graphical analysis of 235 macroscopic experimental data. Geochimica et Cosmochimica Acta 72, 45214536. 
237 Fraysse, F., Pokrovsky, O.S., Schott, J. and Meunier, J.-D. (2006) Surface

238 properties, solubility and dissolution kinetics of bamboo phytoliths. Geochimica et 239 Cosmochimica Acta 70, 1939-1951.

240 Greenwood, J.E., Truesdale, V.W. and Rendell, A.R. (2005) Toward an

241 understanding of biogenic-silica dissolution in seawater-An initial rate approach

242 applied between 40 and $90^{\circ} \mathrm{C}$. Aquatic Geochemistry 11, 1-20.

243 Hamiliton, J.P., Brantley, S.L., Pantano, C.G., Criscenti, L.J. and Kubicki, J.D. (2001)

244 Dissolution of nepheline, jadite and albite glasses: Toward better models for

245 aluminosilicate dissolution. Geochimica et Cosmochimica Acta 65, 3683-3702.

246 Icenhower, J.P. and Dove, P.M. (2000) The dissolution kinetics of amorphous silica

247 into sodium chloride solutions: Effects of temperature and ionic strength.

248 Geochimica et Cosmochimica Acta 64, 4193-4203.

249 Mazer, J.J. and Walther, J.V. (1994) Dissolution kinetics of silica glass as a function

250 of pH between 40 and $85^{\circ} \mathrm{C}$. Journal of Non-Crystalline Solids 170, 32-45.

251 Moya-Laraño, J. and Corcobado, G. (2008) Plotting partial correlation and

252 regression in ecological studies. Web Ecology 8, 35-46.

253 Rickert, D., Schlüter, M. and Wallmann, K. (2002) Dissolution kinetics of biogenic

254 silica from the water column to the sediments. Geochimica et Cosmochimica Acta

255 66, 439-455.

256 Rimstdit, J.D. and Barnes, H.L. (1980) The kinetics of silica-water reactions.

257 Geochimica et Cosmochimica Acta 44, 1683-1699.

258 Rimstidt, J.D. (2015) Rate equations for sodium catalyzed quartz dissolution.

259 Geochimica et Cosmochimica Acta 167, 195-204.

260 Rimstidt, J.D., Brantley, S.L. and Olsen, A.A. (2012) Systematic review of forsterite 261 dissolution data. Geochimica et Cosmochimica Acta 99, 159-178.

262 Seidel, A., Löbbus, M., Vogelsberger, W. and Sonnefeld, J. (1997) The kinetics of

263 dissolution of silica 'Monospher' into water at different concentrations of

264 background electrolyte. Solid State Ionics 101-103, 713-719.

265 Velleman, P.F. and Welsch, R.E. (1981) Efficient computing of regression

266 diagnostics. The American Statistician 35, 234-242.

267 Wirth, G.S. (1980) The heterogeneous kinetics of silica dissolution in aqueous

268 media, Oceanography Ph.D thesis. University of California San Diego, 172 pp.

269 Wirth, G.S. and Gieskes, J.M. (1979) The initial kinetics of dissolution of vitreous

270 silica in aqueous media. Journal of Colloid and Interface Science 68, 492-500. 
271 Xiao, Y. and Lasaga, A. (1996) Ab initio quantum mechanical studies of the kinetics 272 and mechanisms of quartz dissolution: $\mathrm{OH}^{-}$catalysis. Geochimica et Cosmochimica 273 Acta 60, 2283-2295.

274 